\title{
Resumption of elective surgery during the COVID-19 pandemic: what lessons can we apply?
}

\author{
Michael T. Olson - Tania Triantafyllou • Saurabh Singhal (D)
}

Received: 13 May 2020 / Accepted: 22 May 2020 / Published online: 5 June 2020

(c) Springer-Verlag GmbH Austria, part of Springer Nature 2020

\section{Dear Editor:}

An acute respiratory disease caused by the novel coronavirus (SARS-CoV-2), coronavirus disease 2019 (COVID-19), has rapidly spread worldwide, necessitating proactive management decisions from clinicians and centers in an effort to mitigate the risk of infection. In order to focus local resources toward both urgent and non-urgent medical treatment of the overwhelming number of infected patients, while supporting the safety of patients and healthcare staff, elective surgeries have largely been postponed or cancelled. While surgical care can be safely deferred in patients with non-life-threatening conditions, this remains an issue for patients with an illness that significantly impacts quality of life who would otherwise benefit from an elective or semi-elective procedure. Although dependent on location, global rates of COVID-19 are expected to reach their peak within the next few weeks, while the infection is expected to stay in the community for the foreseeable future. Amid strong efforts to control disease spread, and with no predictable end in sight, there is an emphasis on safely while preparing for the resumption of elective operations to meet the surgical needs of more patients. This focus begs several questions, such as, when is

\section{T. Olson, BS}

University of Arizona College of Medicine-Phoenix Campus, Phoenix, AZ, USA

T. Triantafyllou, MD

Hippocration General Hospital of Athens, University of Athens, Athens, Greece

\section{S. Singhal, MBBS, MS (殴}

Department of GI Surgery and Liver Transplantation, Indraprastha Apollo Hospital, New Delhi, India drsaurabhsinghal@gmail.com it appropriate to return to a normal-capacity surgical practice? Are there potential risks with endoscopy or laparoscopy? What challenges exist with preventive measures, and what can be done to prioritize vulnerable patients?

The strategies to prevent viral transmission have been well established in the literature and mainstream media: proper hygiene, self-isolation, and social distancing are paramount. However, these strategies do not reduce the absolute number of infections, they merely lengthen the time it takes for individuals to contract the infection. Although general consensus suggests that a peak will soon be reached for the COVID-19 pandemic, there are major concerns regarding the threat of a viral resurgence if infectioncontrol measures are not meticulously preserved, or if a vaccine is not developed and widely distributed. We can rely on the lessons we have learned in facing the previous severe acute respiratory syndrome (SARS) epidemic in February 2003. In Toronto, Canada, many perceived the SARS outbreak to be over in May 2003, and hospitals relaxed their infection-control precautions. These changes preceded a surge of respiratory illnesses, particularly among hospital employees [1]. The decision of when to return to full capacity will certainly differ based on where an institution is situated relative to the curve of the disease as well as the availability of personal protective equipment (PPE), intensive care unit beds, respirators, and personnel. The goal to optimally provide safe and high-quality surgical care has not wavered, but in resuming elective surgery, it remains vitally important to be considerate of its risks. The American College of Surgeons has since outlined 10 issues to be addressed locally before reinstituting elective surgery (Table 1, adapted from the American College of Surgeons), while the Royal College of Surgeons have also provided updated clinical guidance for surgeons. Addressing these issues 
Table 1 Local resumption of elective surgery guidance ${ }^{\mathrm{a}}$ COVID-19 awareness

Understand the local burden of COVID-19 in one's community, including prevalence and incidence rates, and local isolation mandates

Remain aware of subsequent waves of COVID-19 infection that could require re-entry into the mitigation phase

Understand local COVID-19 diagnostic testing capabilities, and develop testing policy for patients and health care staff

Consider the effect of false-negative test rates (as high as $30 \%$ ), and establish postoperative testing to rule out COVID-19 in symptomatic patients Preparedness

Ensure quality and quantity assessment of local PPE availability, and closely follow PPE recommendations for COVID-19+ patients, patients under investigations, and non-COVID-19 patients

Re-evaluate health care facility capacity, including resources (e.g., beds, ICUs, ventilators), and expansion strategies

Operating rooms should take inventory of existing surgical and cleaning supplies before re-activating elective surgeries

Ensure coordination among surgery, anesthesia, nursing, engineering, housekeeping, and other hospital staff or specialties involved in multidisciplinary care; assure adequate staff volume

Assign a governance committee to clarify, interpret, and iterate policies, make real-time decisions, and initiate and communicate messaging

Patient issues

Consider implementing a multidisciplinary committee to organize patient-surgeon messaging and communication

Develop collaborative process to identify a framework for prioritization of surgical procedures; include input from surgery, anesthesia, nursing, and others

Delivery of safe and high-quality care

Ensure safe, high-quality, high-value care of the surgical patient across the five phases of care continuum

adapted from the American College of Surgeons, "Local Resumption of Elective Surgery Guidance" (2020 Apr 17) and Royal College of Surgeons, "Updated intercollegiate general surgery guidance on COVID-19" (2020 May 30). Decisions regarding elective surgery may be altered based on COVID-19 burden at respective institutions, availability of hospital resources (ICU beds, ventilators, clinicians, PPE), and the COVID-19 trajectory within the hospital

may guide individual timelines of deciding when it is appropriate to return to full capacity.

Although leading surgical societies have guided surgeons in terms of appropriate surgical practice amid the ongoing viral pandemic, certain questions remain, particularly pertaining to the safety of performing minimally invasive surgery in the setting of COVID-19. Concerns have risen from theories that the environment created in a pneumoperitoneum for laparoscopy creates a relatively stagnant volume of gas in the abdominal cavity, which may allow for a concentrated aerosolization of the virus. These concerns have persisted after SARS-CoV-2 viral RNA was identified in the stool of infected patients, with evidence that the virus may be found in the gastrointestinal mucosa [2, 3]. However, infectious SARS-CoV-2 virus has not been isolated from stool samples, despite high viral RNA concentrations [4]. These observations spawn questions regarding the risks involved in gastrointestinal endoscopy. As it pertains to surgical interventions, it would seem that the benefit of performing laparoscopy during the pandemic would outweigh any supposed risk, as minimally invasive surgery would (1) contribute to reduced lengths of stay and faster recovery times, (2) minimize the need for medical treatments, and (3) maximize availability of beds and other crucial resources. Vigneswaran et al. [5] conducted a review of current scientific knowledge regarding viral transmission during laparoscopic and open surgery, finding no evidence to support performing open surgery over laparoscopic to reduce viral transmission. Laparoscopy seems to convey a negligible risk, but nonetheless, all pneumoperitoneum should be safely evacuated from the port attached to a filtration device before closure, trocar removal, specimen extraction, or conversion to open surgery [6].

Depending on local diagnostic testing policies, rapid testing for SARS-CoV-2 infection through realtime reverse transcription polymerase chain reaction (RT-PCR) may be considered for all patients undergoing planned surgery. Nevertheless, there have unfortunately been high false-negative rates reported with RT-PCR testing for SARS-CoV-2 in hospitalized patients clinically diagnosed with COVID-19 [7]. Additionally, some have speculated that the availability of diagnostic tests may actually decrease as community testing demands increase. For these reasons, and in the absence of credible, evidence-based guidelines supporting the contrary, it remains prudent for all surgical staff to wear additional PPE during elective operations, whether open, laparoscopic, or robotic. Wearing additional PPE does not come without challenges to the surgeon, however, as it may restrict mobility, limit peripheral vision, and with headgear, make it challenging to operate amongst other staff, particularly in the deep abdomen. There are logistical delays that are often forgotten when utilizing additional PPE and infection-control measures. When elective surgeries are reinstated, it may be difficult to sustain a reliable supply chain with adequate inventory of PPE for both airborne and droplet/ contact precautions. Operating room staff must also be trained in appropriate donning and doffing, if not done so already, while there are additional technical and administrative measures required to set-up a negative-pressure operating room. These immoderate delays may require significant pre-planning and coordination among all surgical staff. It remains to be determined how these infection-control measures, albeit contributing to the safety of the patient and staff, impact surgical care when elective surgeries are again performed.

At the advent of resuming elective surgeries, it remains a judicious practice to prioritize the surgical care of the most vulnerable populations, such as cancer patients. The American College of Surgeons has recommended use of the Elective Surgery Acuity Scale, which aids the surgical decision-making process of triaging non-emergency operations. On this 
scale, most cancers in highly symptomatic patients are classified with the highest acuity. A recent multicenter study including 105 cancer patients and 536 age-matched non-cancer patients confirmed with COVID-19 found higher risks for severe outcomes among infected cancer patients; notably, patients undergoing cancer surgery also have higher death rates and a greater chance of developing critical symptoms secondary to COVID-19 [8]. Maintaining high surgical quality standards, especially through enhanced recovery programs (ERPs) after cancer surgery, will be paramount in assuring that in-hospital stays are minimized and recovery is facilitated [9]. Doussot et al. [10] recently shared the challenges facing ERPs in the midst of staffing issues. Thus, updating guidelines to help tailor ERPs to the current pandemic situation may be valuable, especially when balancing a schedule with elective surgeries.

Amid strong efforts to control the COVID-19 pandemic, there is anticipation among the surgical community to resume elective operations. Lessons from past epidemics should serve as a reminder to maintain infection-control measures in elective surgery. Considerations of the risk-benefit ratio suggests laparoscopy should be performed in elective surgery when appropriate, with specific adherence to surgical society guidelines. All surgical staff must communicate and prepare for the obstacles that accompany a resumption of elective surgery. Lastly, it remains important to prioritize vulnerable cancer patients, and ERPs should be tailored to maximize their utility amidst the COVID-19 pandemic. The discussion presented herein may serve as an important basis for future exchanges on the topic of elective surgery amidst a global pandemic.

Funding This research did not receive any specific grant from funding agencies in public, commercial, or non-forprofit sectors.

Author Contribution Each individual listed as an author in this manuscript contributed substantially and in accordance with the guidelines of the International Committee of Medical Journal Editors.

Conflict of interest M.T. Olson, T. Triantafyllou, and S. Singhal have no conflicts of interest or financial ties to disclose.

\section{References}

1. Ofner-Agostini M, Wallington T, Henry B, et al. Investigation of the second wave (phase 2) of severe acute respiratory syndrome (SARS) in Toronto, Canada. What happened? Can Commun Dis Rep. 2008;34(2):1-11.

2. Xiao F, Tang M, ZhengX, Liu Y, LiX, Shan H. Evidence for gastrointestinal infection of SARS-CoV-2. Gastroenterology. 2020; https://doi.org/10.1053/j.gastro.2020.02.055.

3. Gu J, Han B, Wang J. COVID-19: gastrointestinal manifestations and potential fecal-oral transmission. Gastroenterology. 2020; https://doi.org/10.1053/j.gastro.2020.02. 054.

4. Wölfel R, Corman VM, Guggemos W, et al. Virological assessment of hospitalized patients with COVID-2019. Nature. 2020; https://doi.org/10.1038/s41586-020-2196-x.

5. Vigneswaran Y, Prachand VN, Posner MC, Matthews JB, Hussain M. What is the appropriate use of laparoscopy over open procedures in the current COVID-19 climate? J Gastrointest Surg. 2020; https://doi.org/10.1007/s11605020-04592-9.

6. Francis N, Dort J, Cho E, et al. SAGES and EAES recommendations for minimally invasive surgery during COVID19 pandemic. Surg Endosc. 2020; https://doi.org/10.1007/ s00464-020-07565-w.

7. Li Y, Yao L, Li J, et al. Stability issues of RT-PCR testing of SARS-CoV-2 for hospitalized patients clinically diagnosed with COVID-19. J MedVirol. 2020; https://doi.org/10.1002/ jmv.25786.

8. Dai M, Liu D, Liu M, et al. Patients with cancer appear more vulnerable to SARS-COV-2: a multi-center study during the COVID-19 outbreak. Cancer Discov. 2020; https://doi.org/ 10.1158/2159-8290.CD-20-0422.

9. Triantafyllou T, Olson MT, Theodorou D, Schizas D, Singhal S. Enhanced recovery pathways vs standard care pathways in esophageal cancer surgery: systematic review and meta-analysis. Esophagus. 2020;17(2):100-12. https://doi. org/10.1007/s10388-020-00718-9.

10. Doussot A, Heyd B, Lakkis Z. We asked the experts: How do we maintain surgical quality standards for enhanced recovery programs after cancer surgery during the COVID19 outbreak? World J Surg. 2020; https://doi.org/10.1007/ s00268-020-05546-7.

Publisher's Note Springer Nature remains neutral with regard to jurisdictional claims in published maps and institutional affiliations. 